\title{
Editorial for the special issue technology for disability sport
}

\author{
Andrey Koptyug ${ }^{1} \cdot$ Brendan Burkett $^{2}$
}

Published online: 1 July 2016

(c) International Sports Engineering Association 2016

Major sporting events, including the Olympic Games, are routinely "brought to our homes" by modern mass media. The Paralympic Games follow the Olympic Games, and are another major competition event. With each summer and winter Paralympic games, such as the 2012 London and the 2014 Sochi Paralympic games, the elite sporting achievements may be new to many of us. The athletic skill and persistent determination drive Paralympic sports. A common thread with the Paralympic athlete is the reliance on some form of technology to compete on the world stage.

Engineering and technology have become an essential part of modern-day sports. This partnership is necessary for coach and athlete as they prepare for, and participate in competitions. From the audience perspective, sports technology can provide a new perspective and insight into the competition and its highlights. The quality of equipment, garments and footwear, and their interaction with athlete's body become an integrated part of the winning strategy in modern sports.

For the athlete with a disability, engineering and technology has always been an essential partnership. Without the assistive devices, the disabled athlete is not able to prepare and participate in sport. Equipment design for disabled athletes is often more complex and customized than for many other applications.

Andrey Koptyug

andrei.koptioug@miun.se

1 Department of Quality Technology, Mechanical Engineering and Mathematics, Sports Tech Research Centre, Mid Sweden University, Akademigatan 1, 83125 Östersund, Sweden

2 School of Health and Sport Sciences, Faculty of Science, Health, Education and Engineering, University of the Sunshine Coast, 90 Sippy Downs Drive, Sippy Downs, Queensland, Australia
The fundamental principles for the use of equipment in Paralympic Sport are safety, fairness, universality and physical prowess. While the safety criterion is relatively straightforward, fulfilling other three can be challenging for the designers and classifiers. It is often impossible to show if the performance of an athlete with a particular assistive device is determined by the athlete rather than by "the impact of technology and equipment". By working together with all parties the sports engineering community can provide affordable safe and reliable assistive devices, technologies for training and rehabilitation, and an improved basis for objective classification.

Research papers in this issue deal with a wide variety of subjects and are multidisciplinary. Four papers in this special issue are related to the athlete and equipment interaction, which is important in all sports but needs special care and precision when working with of paraathletes. The remaining five papers are related to issues important for the training and classification.

We hope that these contributions will improve the integration between athlete and equipment, will provide a better basis for proper classification of the equipment and assistive devices, and, according to the International Paralympic Committee vision, will "enable para-athletes to achieve sporting excellence and inspire and excite the world". 